\title{
Modelling the effect of structure and base sequence on DNA molecular electronics
}

\author{
M. M. D. Ramos and H. M. G. Correia \\ Departamento de Física, Universidade do Minho, Campus de Gualtar, \\ 4710-057 Braga, Portugal
}

\begin{abstract}
DNA is a material that has the potential to be used in nanoelectronic devices as an active component. However, the electronic properties of DNA responsible for its conducting behaviour remain controversial. Here we use a self-consistent quantum molecular dynamics method to study the effect of DNA structure and base sequence on the energy involved when electrons are added or removed from isolated molecules and the transfer of the injected charge along de molecular axis when an electric field is applied. Our results have shown that the DNA molecules of poly $(\mathrm{C})-\mathrm{poly}(\mathrm{G})$ on $\mathrm{B}$ form and poly(A)-poly(T) on A-form have the highest energy released when one electron is added or removed from them and their Z-form has the lowest energy released. Besides, when an electric field is applied to a charged DNA molecule along its axis there is electron transfer through the molecule, regardless of the number and sign of the injected charge, the molecular structure and the base sequence. Results from these simulations provide useful information that is hard to obtain from the experiments and needs to be considered for a further modelling aiming to improve charge transport efficiency in nanoelectronic devices based on DNA.
\end{abstract}

Keywords: Atomistic modelling, double-stranded DNA, injected charge distribution, electric field effect, electron transfer.

PACS numbers: 87.14.Gg; 31.15.Ne; 87.15.Aa; 85.65.th; 36.20.Fr; 87.50.Rr

\section{Introduction}

The molecule of Deoxyribonucleic Acid (DNA) is not only of great biological importance, because it contains the codified genetic information of the live species, but it is also highly interesting under the technological point of view because of its ability of self-assembly and electrical properties.

The electronic processes related with charge injection and transfer in DNA molecules are very important either in biological contexts (e.g. the detection and repairing of damage bases [1]), either in technological contexts (e.g. the use DNA in electrochemical sensors [2, 3] and in future nanotechnologies, such as molecular electronics [4, 5]). Motivated by these potential applications, a large number of theoretical and experimental studies have been carried out on the charge transport properties of DNA, which has been recently reviewed by Taniguchi and Kawai [6].

In spite of all this intense theoretical and experimental effort, the effects of molecular structure and base sequence on the properties of DNA, which affects both the charge transfer through an individual DNA molecule and between the electrodes and the molecule in single molecule electronic devices, are still far from being well understood. In this work, we use a self-consistent quantum molecular dynamics method to understand the physical behaviour of isolated double-stranded DNA molecules of poly(A)-poly(T) and poly(C)-poly $(G)$, with three different molecular structures (A-form, B-form and Z-form), when positive and negative charge injection occurs and the effect of an uniform applied electric field on the injected charge.

\section{Theoretical methods}

\subsection{Self-consistent quantum molecular dynamics method}

In the present work we seek the application of simultaneous self-consistent electronic structure calculations and molecular dynamics to the study of the processes related with charge (electrons and holes) injection and charge transfer in large double-stranded DNA molecules. The electronic structure calculations were performed using a semi-empirical molecular orbital method, which works at CNDO (Complete Neglect of Differential Overlap) level [7, 8] within a cluster model framework. A molecular dynamical method is employed in parallel to perform geometry optimization using the selfconsistent atomic forces obtained from the electronic calculations. 
This strategy is implemented in the CHEMOS code $[9,10]$, which has also the possibility of studying the behaviour of charged DNA molecules under the effect of external applied electric fields. It has the advantage of employing a well-known molecular orbital method, which has been widely tested and validated for a large variety of organic materials, to study the static and dynamic properties of large double-stranded DNA molecules without excessive computational effort.

In the calculations reported here, the CNDO method is described as a semi-empirical application of the unrestricted Hartree-Fock method using a linear combination of Slater-type atomic orbitals, the $\mathrm{CNDO} / 2$ parameters (electronegativity, bonding parameter) and Slater orbital exponents selected by Pople and Beveridge [7] and the basis set for the elements of first, second and third row of the Periodic Table are s, sp and spd, respectively.

\subsection{The method for charge distribution analysis}

The charge distribution in the system is obtained using the linear combination of atomic orbitals (LCAO) approximation. Within unrestricted CNDO method, the total electronic charge in the system is given by

$$
N^{\alpha}+N^{\beta}=\sum_{\mu v}\left(P_{\mu v}^{\alpha}+P_{\mu v}^{\beta}\right) S_{\mu v}
$$

where $N^{\alpha}$ and $N^{\beta}$ are the total number of spin-up and spin-down valence electrons, $P_{\mu \nu}^{\alpha}$ and $P_{\mu \nu}^{\beta}$ are elements of the electronic density matrix associated with spin-up and spin-down electrons and $S_{\mu \nu}$ is the overlap integral matrix. The electronic charge can be split into contributions associated with the various atomic orbitals. Using the Mulliken population analysis [11], the charge on atom A is then be given by

$$
q_{A}=n_{A}^{0}-\sum_{v} P_{v v}^{A}
$$

where $n_{A}^{0}$ is the number of valence electrons within an isolated neutral atom $\mathrm{A}$ and $v$ runs over the all occupied orbitals of atom A.

\subsection{Methods for calculating ionization potential and electron affinity}

The first ionization potential (IP) (the energy needed to remove an electron or add a hole to the neutral system) and electron affinity (EA) (the energy gained on adding an electron to the neutral system) will be calculated by three different methods.

The adiabatic ionization potential (AIP) and electron affinity (AEA) are obtained from the energy differences between the optimized geometry of neutral and charged systems

$$
\begin{aligned}
& A I P=E_{Q=+1}-E_{Q=0} \\
& A E A=E_{Q=0}-E_{Q=-1}
\end{aligned}
$$

where $E_{Q}$ is the internal energy of the system with optimized geometry and total charge $Q$.

The vertical ionization potential (VIP) and electron affinity (VEA) are defined as the difference in energy between the neutral and the charged systems at the geometry of the neutral.

$$
\begin{gathered}
V I P=E_{Q=+1}^{\text {geom } Q=0}-E_{Q=0} \\
V E A=E_{Q=0}-E_{Q=-1}^{\text {geom } Q=0}
\end{gathered}
$$

If one assumes no change of the other orbitals on ionization, Koopmans theorem [12] states that

$$
K I P=-E_{\text {номо }}
$$

where KIP is the ionization potential associated with the removal of an electron from the highest occupied molecular orbital with energy $E_{\text {Номо }}$. If we also ignore orbital re-organization when one electron is add to the neutral system, we can write

$$
K E A=-E_{\text {LUMO }}
$$

where KEA is the electron affinity associated with adding an electron to the lowest unoccupied molecular orbital with energy $E_{L U M O}$. 


\section{Results}

Theoretical and experimental studies shown that double-stranded DNA has structural polymorphism [13]. Generally in water solution DNA molecules assumes the B-form. However, DNA molecules can assume the A-form, when there is little water available to interact with the double-stranded, or the Zform, when the water solution has a high concentration of positive ions.

\subsection{Testing the reliability of the self-consistent approach based on the CNDO method}

The self-consistent quantum-chemical molecular dynamics approach (see section 2.1) was developed for systems for which atomic positions and electronic structures are coupled problems. Successful application of this approach has been demonstrated for theoretical studies of charge-induced defects (localized charge dressed with lattice distortion) in conjugated polymers and their mobility along the polymer strands $[10,14]$. However, its validity and accuracy have still to be shown in predicting the molecular properties of DNA in neutral and charge states. To have confidence in the CNDO method with minimal basis set as a correct description of those properties, we need to investigate how well it predicts the electronic structure (the energies of the highest occupied molecular orbital $\left(\mathrm{E}_{\mathrm{HO} о}\right)$ and lowest unoccupied molecular orbital $\left(\mathrm{E}_{\mathrm{LUMO}}\right)$ ), the electronic properties (ionization potential and electron affinity) and the charge distribution in DNA fragments. The best way of testing the reliability of a method is to compare its results with the corresponding experimental data whenever available.

We will first test the performance of our theoretical method in connection with atomic charge distribution within a nucleotide by calculating the charge distribution of deoxycytidine5 'monophosphate (5'dCMP) molecule and comparing our results with the atomic charges obtained from X-ray data [15] and those calculated by the method of CNDO/2 using the spd-basis set [16].

In comparing the 5'dCMP atomic charges calculated by us and those reported elsewhere [15, 16], we found a good correlation between our results and both experimental and theoretical data. The table 1 shows the total charges belonging to 5'dCMP fragments as revealed by the experiment [15] and calculated by us and by the CNDO/2 (spd-basis set) technique [16]. We should note that this charge separation by the molecular fragments has been done only after the calculation of the atomic charges for the complete molecule. The agreement between our results and those obtained from X-ray data is not so good as the one revealed by the $\mathrm{CNDO} / 2$ (spd-basis set) technique, but the concordance in the trend is completely reproduced.

Table 1. Total charges for 5'dCMP fragments.

\begin{tabular}{cccc}
\hline & \multicolumn{3}{c}{ Total charge (electrons) } \\
\cline { 2 - 4 } & Experiment [15] & CNDO/2 (spd-basis set) [16] & $\begin{array}{c}\text { Our } \\
\text { calculations }\end{array}$ \\
\hline Cytosine. $\mathrm{H}^{+}$ & 0.968 & 0.722 & 0.679 \\
Deoxyribose & 0.327 & 0.302 & 0.270 \\
Phosphate & -1.294 & -1.024 & -0.949 \\
\hline
\end{tabular}

Despite the dependence of the Mulliken atomic charges on the method and bases set used, Mulliken population analysis can provide a valuable interpretation of charge distribution within a DNA molecule.

In order to assess the performance of our theoretical method for describing the electronic properties of DNA, we start by comparing IPs and EAs of bases (see table 2) and N-methylated nucleobases (see table 3) with experimental data available.

The comparison between our results and the experimental ones for DNA bases permits us to conclude that all the methods used to calculate IPs and EAs predict the same trends for IPs but not for EAs. The calculated AIPs and AEAs are in better agreement with the experimental values, but the concordance in the trend is not completely reproduced for cytosine, which AIP and AEA are smaller than those of adenine. The negative EAs from these studies are in agreement with the experimental results obtained using low-energy electron transmission spectroscopy (ETS) [17] and the VEAs determined at density 
functional level, using different exchange correlation functionals and basis sets $[18,19]$. However, the magnitude of AIPs are overestimated and AEAs are underestimated.

Table 2. Comparison between experimental and calculated first ionization potentials (AIP, VIP, KIP) and electron affinities (AEA, VEA, KEA) of DNA bases.

\begin{tabular}{|c|c|c|c|c|c|c|c|c|}
\hline \multirow{2}{*}{ DNA bases } & \multicolumn{4}{|c|}{ Ionization potentials $(\mathrm{eV})$} & \multicolumn{4}{|c|}{ Electron affinities $(\mathrm{eV})$} \\
\hline & AIP & VIP & KIP & Exp. [20] & AEA & VEA & KEA & Exp. [17] \\
\hline Guanine (G) & 8.09 & 8.48 & 9.83 & 7.77 & -1.33 & -3.12 & -3.05 & -0.46 \\
\hline Adenine (A) & 8.91 & 9.48 & 11.00 & 8.26 & -1.21 & -1.43 & -3.21 & -0.54 \\
\hline Cytosine (C) & 8.62 & 9.33 & 10.92 & 8.68 & -1.22 & -1.64 & -2.27 & -0.32 \\
\hline Thymine (T) & 9.05 & 9.60 & 11.76 & 8.87 & -0.49 & -0.94 & -2.06 & -0.29 \\
\hline
\end{tabular}

Table 3. Comparison between experimental and calculated first ionization potentials (AIP, VIP, KIP) and electron affinities (AEA, VEA, KEA) of N-methylated nucleobases.

\begin{tabular}{llllllllll}
\hline & \multicolumn{3}{c}{ Ionization potentials (eV) } & & \multicolumn{3}{c}{ Electron affinities (eV) } \\
\cline { 2 - 4 } & AIP & VIP & KIP & Exp. [21] & & AEA & VEA & KEA \\
\hline Guanine (G) & 8.04 & 8.41 & 9.84 & 8.24 & & -1.27 & -3.03 & -2.95 \\
Adenine (A) & 8.37 & 8.77 & 10.93 & 8.44 & & -1.98 & -2.22 & -3.18 \\
Cytosine (C) & 8.39 & 8.75 & 10.74 & 8.94 & & -0.71 & -1.03 & -2.16 \\
Thymine (T) & 8.91 & 9.46 & 11.31 & 9.14 & & -0.51 & -0.94 & -2.15 \\
\hline
\end{tabular}

The AIP values for N-methylated bases follow the same trend as the experimental ones and they differ by $0.07-0.55 \mathrm{eV}$ from experimental values. The adiabatic method give negative EAs and follow the same trend as the experimental data from ETS measurements [17], although the magnitude of these calculated energies are much less reliable.

Having determined the performance of our method in connection with DNA bases, it is time to assess its performance for evaluating IPs and EAs for small double-strand nucleotides and nucleotide models in different DNA conformations. Since to the best of our knowledge there is no experimental IPs and EAs reported in the literature for such systems, the best way of testing the reliability of the CNDO method used in this work to study the electronic properties of DNA is to compare its results for standard geometries with other theoretical results.

In table 4, IPs and EAs for symmetrical homodimers consisting of two Watson-Crick base pairs (adenosine-thymidine (AT) and guanine-cytidine (GC)) of nucleosides (i.e., bases attached to deoxyribose moieties), stacked over each other to mimic the standard A-DNA and B-DNA conformations, computed within the CNDO approximation are compared with the results estimated from total energies obtained by Starikov with the method PM3-CI [22].

Table 4. Comparison of first ionization potentials and electron affinities of symmetrical homodimers GC-GC and AT-AT in B-form and A-form calculated at CNDO (our work) and PM3-CI [22] levels.

\begin{tabular}{|c|c|c|c|c|c|c|c|}
\hline & & \multicolumn{3}{|c|}{ Ionization potentials $(\mathrm{eV})$} & \multicolumn{3}{|c|}{ Electron affinities $(\mathrm{eV})$} \\
\hline & & \multicolumn{2}{|c|}{ Our Work } & \multirow{2}{*}{$\begin{array}{c}\text { PM3-CI [22] } \\
\text { VIP }\end{array}$} & \multicolumn{2}{|c|}{ Our Work } & \multirow{2}{*}{$\begin{array}{c}\text { PM3-CI [22] } \\
\text { VEA }\end{array}$} \\
\hline & & KIP & VIP & & KEA & VEA & \\
\hline \multirow{2}{*}{ A-form } & AT-AT & 10.15 & 8.87 & 7.66 & -2.54 & -0.92 & 0.42 \\
\hline & GC-GC & 8.99 & 7.66 & 6.89 & -2.58 & -1.08 & 0.93 \\
\hline \multirow{2}{*}{ B-form } & AT-AT & 10.15 & 9.09 & 7.45 & -2.55 & -0.97 & 0.61 \\
\hline & GC-GC & 9.14 & 7.82 & 7.23 & -2.52 & -1.04 & 1.23 \\
\hline
\end{tabular}

The VIP follow the same trends with the exception of the VIP of AT-AT in A-form, which is smaller than AT-AT in the B-form. The VEAs do not follow the same trends nor having the same sign. 
Despite its success in predicting VIP trends for these dinucleoside homodimers, the CNDO method greatly overestimates VIPs whereas PM3-CI [22] gives VIPs for GC-GC and AT-AT in B-form that compares well with ab initio results for dimers of Watson-Crick pairs [23].

We report in table 5 the evolution of IPs and EAs for small sequences of stacked base paired dinucleosides in the B-form.

Table 5. Comparison of IPs and EAs for various stacked base paired dinucleosides in standard B-

DNA conformation.

\begin{tabular}{ccccccc}
\hline & \multicolumn{2}{c}{ Ionization potentials (eV) } & & \multicolumn{2}{c}{ Electron affinities (eV) } \\
\cline { 2 - 3 } \cline { 5 - 6 } \cline { 5 - 6 } & KIP & VIP & & KEA & VEA \\
\hline$(\mathrm{GC})_{2}$ & 9.14 & 7.82 & & -2.52 & -1.04 \\
$(\mathrm{GC})_{3}$ & 9.03 & 7.69 & & -2.48 & -0.95 \\
$(\mathrm{GC})_{4}$ & 8.97 & 7.62 & & -2.47 & -0.95 \\
\hline$(\mathrm{AT})_{2}$ & 10.15 & 9.09 & & -2.55 & -0.97 \\
$(\mathrm{AT})_{3}$ & 10.06 & 8.94 & & -2.53 & -0.99 \\
$(\mathrm{AT})_{4}$ & 9.95 & 8.49 & & -2.30 & -0.85 \\
\hline
\end{tabular}

Although in qualitative agreement with $a b$ initio calculations at Hartree-Fock and Density Functional levels [23], our VIP and KIP values for both $(\mathrm{AT})_{\mathrm{n}}$ and $(\mathrm{GC})_{\mathrm{n}}$ are overestimate.

Having determined the performance of our CNDO method in connection with molecular conformation and base sequence on dinucleoside dimmers and various stacked based paired dinucleosides, it is time to assess its performance with respect to the effect of sugar-phosphate backbones on the electronic properties of stacked DNA base pairs. Table 6 gives the calculated IPs and EAs for double-stranded oligonucleotides with small $(\mathrm{GC})_{\mathrm{n}}$ and $(\mathrm{AT})_{\mathrm{n}}$ pairs in the standard A- and B-DNA conformations.

Table 6. The first ionization potentials and electron affinities for various stacked Watson-Crick pairs including the backbones composed of sugars and phosphates in standard A- and B-DNA conformations.

\begin{tabular}{|c|c|c|c|c|c|}
\hline & \multicolumn{2}{|c|}{ Ionization potentials $(\mathrm{eV})$} & \multicolumn{2}{|c|}{ Electron affinities $(\mathrm{eV})$} \\
\hline & & KIP & VIP & KEA & VEA \\
\hline \multirow{6}{*}{ A-form } & $(\mathrm{GC})_{2}$ & 7.06 & -3.87 & 5.71 & 14.96 \\
\hline & $(\mathrm{GC})_{3}$ & 7.09 & -7.49 & 5.92 & 17.68 \\
\hline & $(\mathrm{GC})_{4}$ & 7.46 & -12.92 & 6.52 & 22.06 \\
\hline & $(\mathrm{AT})_{2}$ & 6.80 & -3.97 & 5.94 & 16.28 \\
\hline & $(\mathrm{AT})_{3}$ & 6.71 & -6.32 & 6.06 & 17.47 \\
\hline & $(\mathrm{AT})_{4}$ & 6.68 & -12.32 & 6.18 & 23.88 \\
\hline \multirow{6}{*}{ B-form } & $(\mathrm{GC})_{2}$ & 9.29 & 0.74 & 6.64 & 14.32 \\
\hline & $(\mathrm{GC})_{3}$ & 9.54 & -6.38 & 8.51 & 19.65 \\
\hline & $(\mathrm{GC})_{4}$ & 9.89 & -9.90 & 8.96 & 20.94 \\
\hline & $(\mathrm{AT})_{2}$ & 7.55 & 1.31 & 6.23 & 17.66 \\
\hline & $(\mathrm{AT})_{3}$ & 8.54 & -2.32 & 7.39 & 22.50 \\
\hline & $(\mathrm{AT})_{4}$ & 8.37 & -9.26 & 7.49 & 25.50 \\
\hline
\end{tabular}

For both $(\mathrm{AT})_{\mathrm{n}}$ and $(\mathrm{GC})_{\mathrm{n}}$, double stranded oligonucleotides VIP decreases and VEA increases as the number of stacked based paired dinucleotides increases. Although these trends are in agreement with the ones obtained for double-stranded oligonucleosides (see table 5) the changes of VIP and VEA with $n$ is more pronounced. These results suggest that phosphates have a rather large effect on the IPs and EAs of DNA. Significant variations occur for VIP and VEA as compared with KIP and KEA 
because removing or adding an electron from the oligonucleotides leads to a substantial change in orbital re-organization. Therefore, the IP values obtained from Koopmans theorem [24] is in considerable error for double-stranded DNA. Comparing the results of tables 5 and 6 for HOMOLUMO energy gaps, we can also conclude that the phosphates strongly affect the electronic structure of the DNA molecules. Moreover, the HOMO-LUMO energy gap, for the dimmer unit of stacked GC base pairs with backbones in B-form is $1.28 \mathrm{eV}$ larger than the value obtained by Density Functional Theory [25].

The comparison between our results and other theoretical and experimental ones permits us to conclude that generally our method yields correct predictions of atomic charge distributions, ionization potentials and electron affinities for DNA molecules. However, in line with other results from CNDO the magnitude of the energies is much less reliable. Nevertheless, it seems to predict the right trends. Therefore, the CNDO method combined with molecular dynamics seems to be valuable approach for such studies in larger double-stranded DNA molecules.

\subsection{Analysis of the molecular internal energy}

To understand the effects of the three different forms of DNA shown in figure 1 on the properties of two large isolated double-stranded DNA molecules with different base pair sequence, we built molecules of poly(A)-poly(T) and poly(C)-poly $(\mathrm{G})$ with a single pitch and the structures of types A, B and $\mathrm{Z}$.

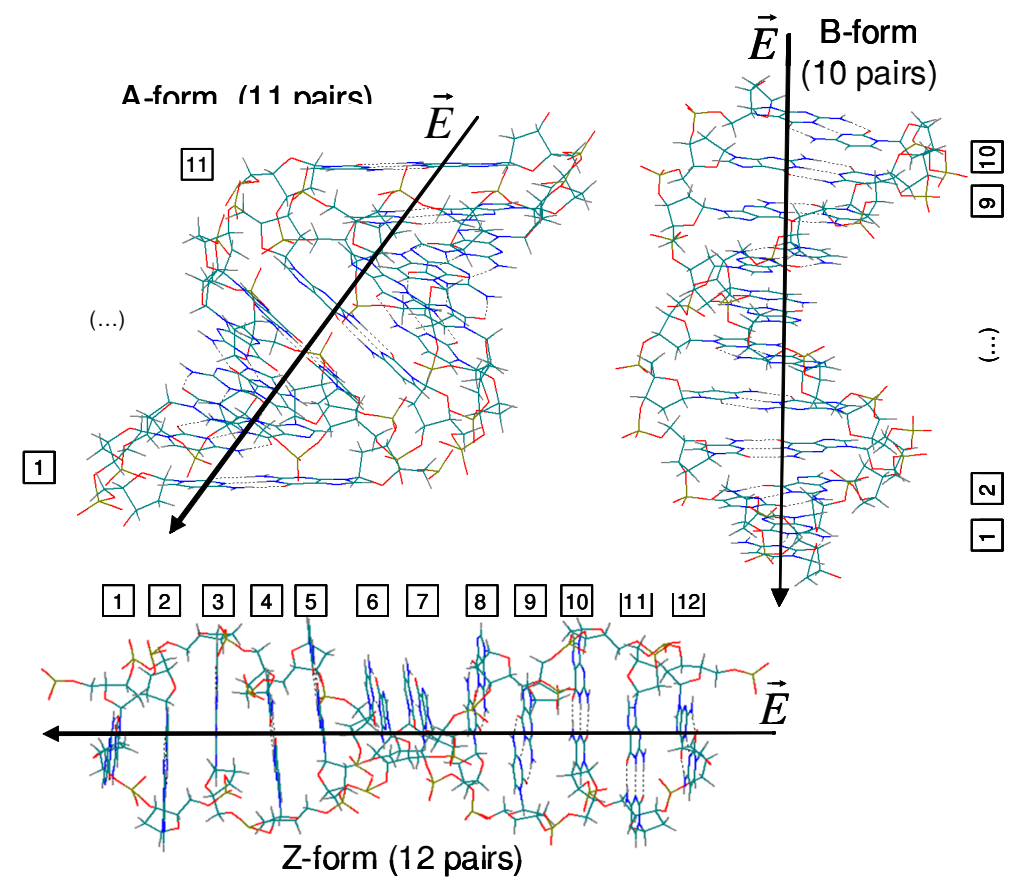

Figure 1 - The types of molecular structure of double-stranded DNA considered in this work. The forms $\mathrm{A}, \mathrm{B}$ and $\mathrm{Z}$ have a single pitch with 11,10 and 12 base pairs, respectively. The arrows indicate the direction of the external electric field $(\vec{E})$ to be applied. The nucleotide pairs (phosphate-sugarpurine-pyrimidine-sugar-phosphate) are numbered for easier identification.

Before charge is injected in these molecules, we got their optimized geometry and we calculated their internal energy and the Mulliken atomic charge distribution throughout the molecule. When one or two electrons are added or removed, we use the optimized structures of the uncharged DNA molecules as a starting point for subsequent geometry optimization of the resulting ions, getting in the end the internal energy and the Mulliken atomic charge distribution of the charged DNA molecules. We found that only tens of molecular dynamics time-steps were needed to obtain the optimized structure of both positive and negative ions. These results indicates that the optimized geometry of 
charged and uncharged DNA molecules do not differ significantly. This behaviour of DNA molecules is similar to what was found for other biological molecules, such as proteins [26].

Figure 2 shows that independently of the structure and the type of base pairs, charged DNA molecules are energetic more stable than uncharged molecules and their stability increases as the ion becomes more negative. Table 7 shows the results obtained for the first ionization potentials and electron affinities of DNA molecules depicted in figure 1 ..

Table 7. The first ionization potentials (AIP, VIP, KIP) and electron affinities (AEA, VEA, KEA) of poly $(\mathrm{C})-\operatorname{poly}(\mathrm{G})$ and poly $(\mathrm{A})-\operatorname{poly}(\mathrm{T})$ shown in figure 1 .

\begin{tabular}{llcccccccc}
\hline & & \multicolumn{3}{c}{ Ionization potentials $(\mathrm{eV})$} & & \multicolumn{3}{c}{ Electron affinities (eV) } \\
\cline { 3 - 5 } \cline { 8 - 9 } & & AIP & VIP & KIP & & AEA & VEA & KEA \\
\hline \multirow{3}{*}{ Poly(C)-poly(G) } & A-form & -47.16 & -12.72 & 6.64 & & 65.94 & 14.97 & 6.33 \\
& B-form & -93.37 & -40.05 & 10.43 & & 107.92 & 54.04 & 9.16 \\
& Z-form & -13.73 & -5.59 & 8.28 & & 26.48 & 17.51 & 6.44 \\
\hline \multirow{3}{*}{ Poly(A)-poly(T) } & A-form & -97.81 & -62.36 & 6.54 & & 109.91 & 70.57 & 6.13 \\
& B-form & -53.65 & -36.31 & 6.14 & & 55.98 & & 41.36 & 5.82 \\
& Z-form & -30.14 & -21.47 & 6.14 & & 42.54 & 33.85 & 5.59 \\
\hline
\end{tabular}

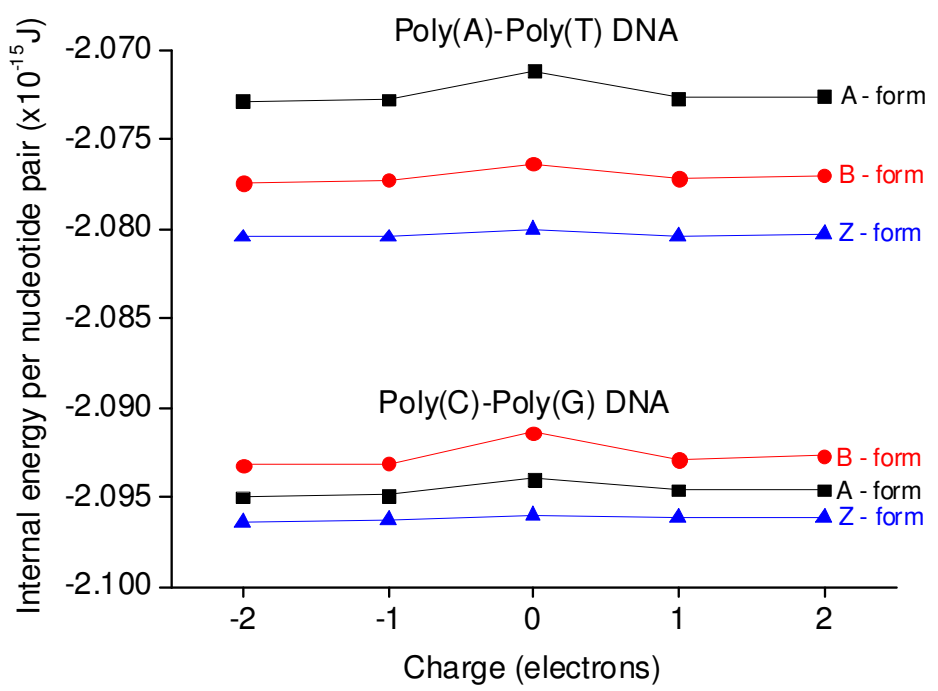

Figure 2 - The internal energy of the DNA fragments shown in figure 1 as a function of the molecular charge.

The analysis of our results for adiabatic and vertical ionization potentials and electron affinities shows interesting features. One is the existence of energy release for the injection of both positive and negative charges. Another important aspect is that, for both base sequence, adding and removing one electron release less energy for the Z-form. One striking result is that the larger energy released due to charge injection occurs for poly $(\mathrm{A})$-poly $(\mathrm{T})$ in the A-form and poly $(\mathrm{C})$-poly $(\mathrm{G})$ in the B-form.

We should note that the calculated electronic structure and the molecular orbitals are strongly dependent on the CNDO parameter used and the basis set of atomic orbitals used to construct the wave function. Therefore, the absolute value of the calculated energies may not be corrected, but we expect the predicted trends for the effect of DNA structure and base sequence to be reliable and these show important features.

\subsection{The distribution of injected charge}

Although Yoo and its coworkers [29] have observed experimentally the semiconducting behaviour of poly(A)-poly(T) and poly $(\mathrm{C})-\operatorname{poly}(\mathrm{G})$ molecules, which are considered synthetic DNA polymers, the 
injected charge distribution in these molecules is completely different from those obtained for semiconducting conjugated polymers [14, 30].

When one add or remove one or two electrons from a DNA molecule, the injected charge is distributed by of all nucleotide pairs and it is not localised over a few neighbouring nucleotide pairs, as was predicted for conjugated polymer molecules [14, 30]. Figure 3 shows that the excess of the injected charge stored in each nucleotide pair varies throughout the molecular axis, regardless the sign and absolute value of the injected charge, the type of structure and the type of base pairs. However, the amount of charge stored in each nucleotide pair and its distribution by the nucleotide fragments of both strands depends on the molecular structure and the type of base pairs.

For both poly(A)-poly(T) and poly(C)-poly $(\mathrm{G})$ the variation of the stored charge between adjacent nucleotides along the molecular axis is less pronounced for the Z-form. The injected charge is mainly stored on the phosphate and sugars of both strands for poly(A)-poly(T) regardless of the DNA structure, whereas in poly $(\mathrm{C})-\operatorname{poly}(\mathrm{G})$ the localization of the excess positive or negative charge depends on the conformation. In this case, the injected charge is mainly stored on the sugars and guanines of poly $(\mathrm{G})$ strand for A-form, on the phosphates and sugars of the same strand for Z-form and for the B-form on the phosphates of both strands as well as on the sugars and guanines of poly $(\mathrm{G})$ strand. The charge storage on the guanine sites are in good agreement with theoretical results of Brunaud et al. [31] when a single hole is delocalized along three different DNA sequences. The charge storage on the sugar-phosphate backbone is in agreement with experimental results of Ray et al. [27].

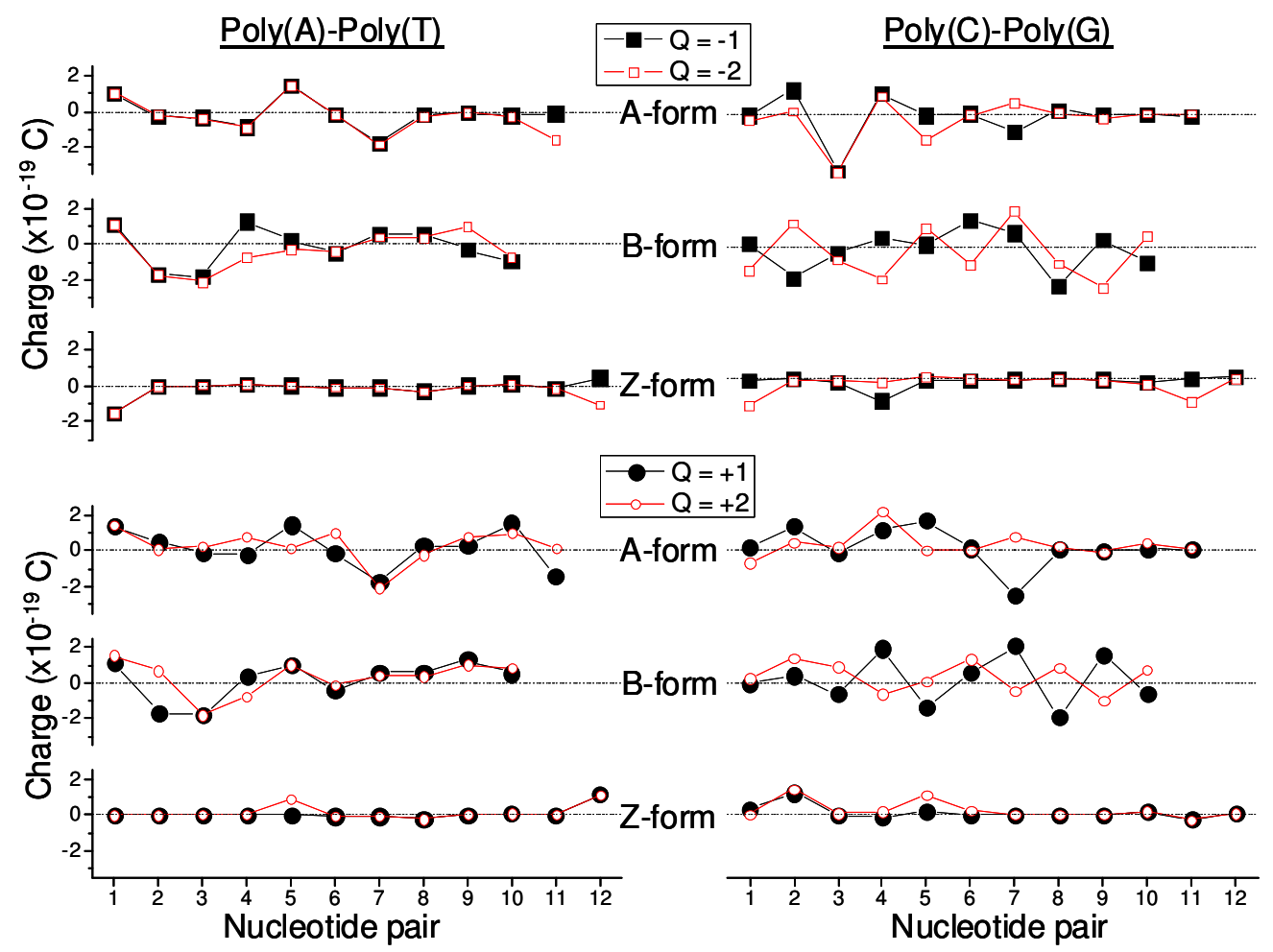

Figure 3 - Changes in the charge of the nucleotide pairs along the molecular axis caused when one and two electrons are added $(\mathrm{Q}=-1, \mathrm{Q}=-2)$ or remove $(\mathrm{Q}=+1, \mathrm{Q}=+2)$ from the DNA molecules shown in figure 1 . The marks indicate the data points that were calculated explicitly, whilst the curves are simply a guide to the eye.

In contrast with conjugated polymers, it is not possible to distinguish from the injected charge distribution in DNA molecules the sign and number of charges that was added to those molecules.

\subsection{The charge-induced distortion}


In order to study the charge-induced distortion in double-stranded DNA molecules, a distortion parameter is defined by:

$$
d_{n}=\sqrt{\left\{\left[\frac{\sum_{i} m_{n, i}\left(x_{n, i}^{Q}-x_{n, i}^{Q=0}\right)}{\sum_{i} m_{n, i}}\right]^{2}+\left[\frac{\sum_{i} m_{n, i}\left(y_{n, i}^{Q}-y_{n, i}^{Q=0}\right)}{\sum_{i} m_{n, i}}\right]^{2}+\left[\frac{\sum_{i} m_{n, i}\left(z_{n, i}^{Q}-z_{n, i}^{Q=0}\right)}{\sum_{i} m_{n, i}}\right]^{2}\right.}
$$

where $m_{n, i}, x_{n, i}^{Q}, y_{n, i}^{Q}, z_{n, i}^{Q}$ are the mass and the coordinates $x, y$ and $z$ of atom $i$ within the nucleotide $n$.

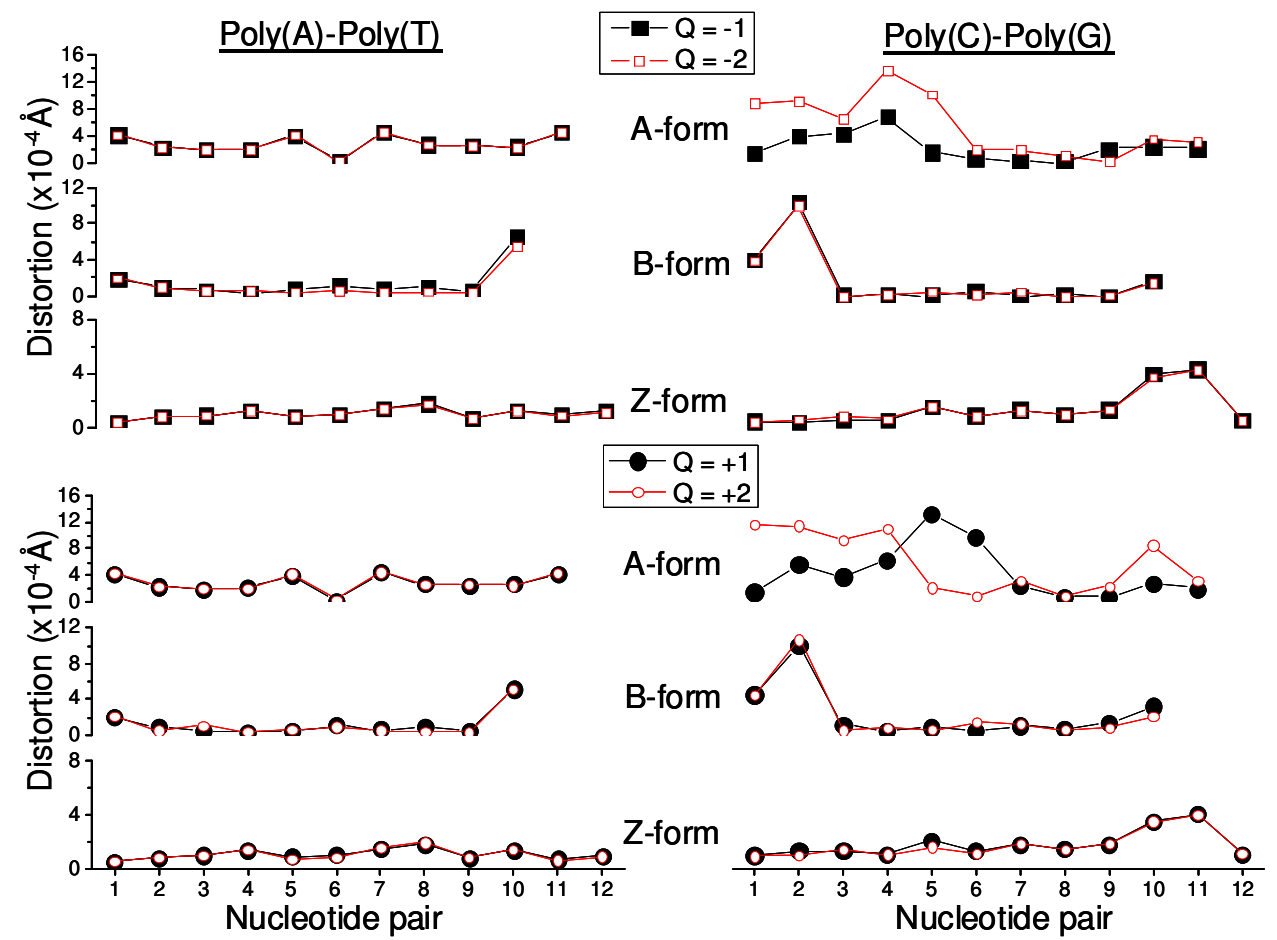

Figure $4-$ Changes in the distortion parameter of the nucleotide pairs along the molecular axis caused when one and two electrons are added $(\mathrm{Q}=-1, \mathrm{Q}=-2)$ or remove $(\mathrm{Q}=+1, \mathrm{Q}=+2)$ from the DNA molecules shown in figure 1 . The marks indicate the data points that were calculated explicitly, whilst the curves are simply a guide to the eye.

Figure 4 shows that the distortion pattern induced by the injected charge varies with the DNA structure and the type of base pairs, but does not change significantly with the number and sign of the injected charges, with the exception of the A-form of poly $(\mathrm{C})-\operatorname{poly}(\mathrm{G})$. Besides, we found no correlation between the distortion pattern (see figure 4) and the distribution of the injected charge per nucleotide pair (see figure 3 )along the molecular axis.

The structures of poly(A)-poly $(\mathrm{T})$ and $\operatorname{poly}(\mathrm{C})-\operatorname{poly}(\mathrm{G})$ nucleotide pairs and the distance between stacked base pairs do not change drastically by charging the molecule. A greater distortion is expected at finite temperature and in aqueous solutions [28, 32]. Nevertheless, our results are a useful approximation of probable behaviour at the temperature of zero Kelvin.

\subsection{The effect of the applied electric field}

When an uniform electric field is applied to a charged DNA molecule along its molecular axis, the end of the molecules on the direction of the field becomes positively charged and the opposite end becomes negatively charged as a result of electron transfer along the molecular axis in opposite direction of the applied field. The amount of positive and negative charge stored at both ends of the DNA molecule increases as the strength of the external applied electric field increases (see figure 5). 
The electron transfer does seem to occur regardless the number and sign of the injected charge on DNA molecules, their structure and type of base pairs.

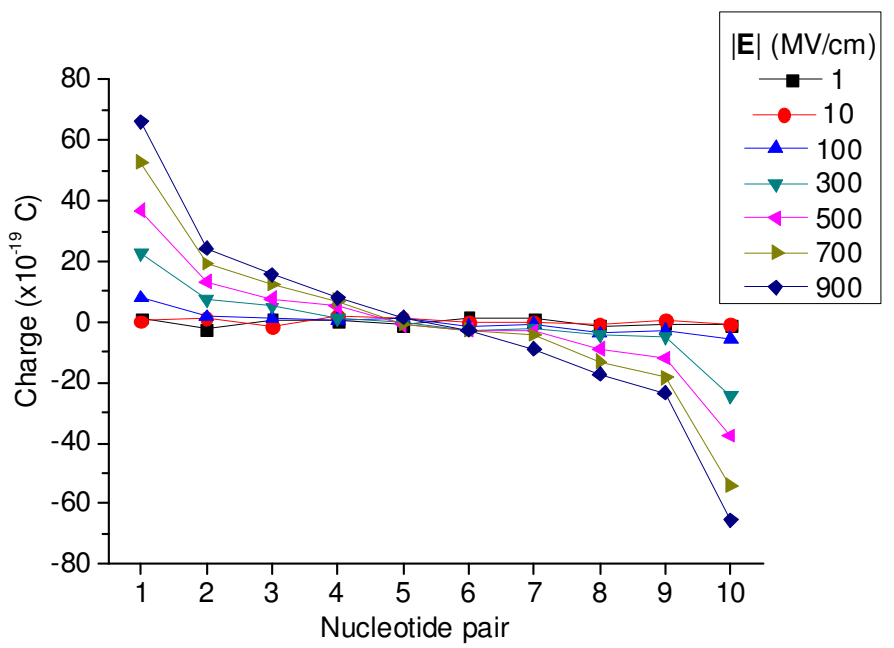

Figure 5 - The effect of the electric field strength (|E|) on the distribution of the injected charge (one electron) into poly $(\mathrm{C})-\operatorname{poly}(\mathrm{G})$ DNA molecule on B-form, for an external applied field along the direction shown in figure 1.

Although the mechanism of charge transfer along the molecular axis of poly(A)-poly(T) and poly(C)poly $(\mathrm{G})$ seems to be similar, the amount of electron transfer for the same applied electric field depends on the molecular structure (see figure 6). However, the amount of electron transfer is similar for poly(A)-poly(T) and poly(C)-poly $(\mathrm{G})$ when both double-stranded DNA molecules adopt the same molecular structure (see figure 6). The relative amount of electron transfer for the three DNA structures considered in this work can be summarized as $\mathrm{A}<\mathrm{B}<\mathrm{Z}$.

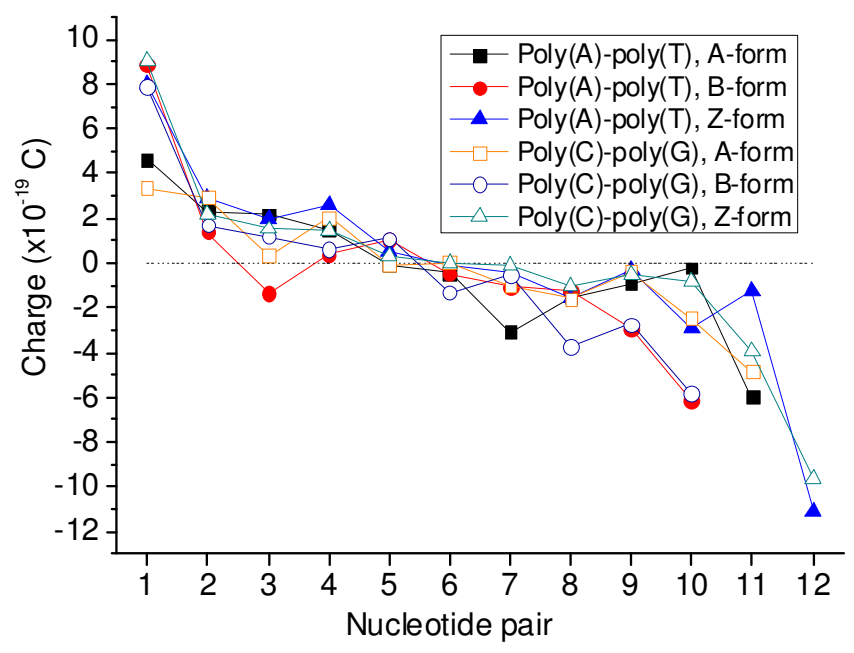

Figure 6 - The effect of the molecular structures' A-form, B-form and Z-form on the distribution of the injected charge (one electron) into poly(A)-poly(T) and poly $(\mathrm{C})-\operatorname{poly}(\mathrm{G})$ DNA molecules, for an external applied electric field of $100 \mathrm{MVcm}^{-1}$ along the direction show in figure 1.

These results suggest that electron transfer through a single double-stranded DNA molecule does not depend on base pair sequence, which is in agreement with recent experiments for a complex sequence [33]. 


\section{Conclusions}

We studied the intramolecular properties involved in the injection of electrons and holes into isolated double-stranded DNA molecules, with three different molecular structures and two different bases pair sequences and the effect of an applied electric field parallel to the molecular axis on the injected charge.

Our results show that the injection of positive and negative charge gives rise to energy release and the energetic stability of the formed ions increases as their charge becomes more negative. This behaviour does not depend on the base sequence and molecular structure. The highest energy released occurs for $\operatorname{poly}(\mathrm{A})-\operatorname{poly}(\mathrm{T})$ in the A-form and $\operatorname{poly}(\mathrm{C})-\operatorname{poly}(\mathrm{G})$ in the B-form and the lowest one when they both have the Z-form. Besides, the excess of electrons or holes are delocalised along the molecular axis and there is no correlation between the distortion and the distribution of the injected charge patterns. When an external electric field is applied along the molecular axis there is electron transfer along the molecular axis and both molecular ends becomes charged of opposite sign, regardless of the number and sign of the injected charge, the molecular structure and the base pair sequence. Moreover, the direction of the electron transfer is opposite to the direction of the applied electric field and the amount of the electron transfer increases as the strength of the applied electric field increases. These results suggest that electron transfer should be the mechanism for intramolecular charge transport in DNA molecules and only the amount of electron transfer seems to depend on molecular structure.

\section{Acknowledgements}

This work is part of the research projects POCTI/CTM/41574/2001 and CONC-REEQ/443/EEI/2005, approved by the Portuguese Foundation for Science and Technology (FCT) and supported by the European Community Fund FEDER. One of us (H.M.G.C.) is also indebted to FCT for financial support under PhD grant no. SFRH/BD/11231/2002.

\section{References}

[1] Endres R G, Cox D L and Singh R R P 2004 Rev. Mod. Phys. 76 195-214.

[2] Drummond T G, Hill M G and Barton J K 2003 Nat. Biotechnol. 21 1192-99.

[3] Kelley S O, Boon E M, Barton J K, Jackson N M and Hill M G 1999 Nucleic Acids Res. 27 4830-37.

[4] Malyshev A V 2007 Phys. Rev. Lett. 98096801 (4pp).

[5] Nogues C, Cohen S R, Daube S, Apter N and Naaman R 2006 J. Phys. Chem. B 110 8910-13.

[6] Taniguchi M and Kawai T 2006 Physica E 33 1-12.

[7] Pople J A and Beveridge D L, Approximate Molecular Orbital Theory, McGraw-Hill, New York, 1970.

[8] Murrell J N and Harget A J, Semi-empirical self-consistent-field molecular orbital theory of molecules, Wiley-Interscience,, London, 1972.

[9] Wallace D S, D. Phil. Thesis, University of Oxford, 1989.

[10] Wallace D S, Stoneham A M, Hayes W, Fisher A J and Harker A H 1991 J. Phys.-Condes. Matter 3 3879-903.

[11] Mulliken R S 1955 J. Chem. Phys. 23 1833-40.

[12] Koopmans T 1934 Physica 1 104-13.

[13] Saenger W, Principles of Nucleic Acid Structure, Springer, New York, 1984.

[14] Correia H M G and Ramos M M D 2005 Comput. Mater. Sci. 33 218-23.

[15] Pearlman D A and Kim S H 1985 Biopolymers 24 327-57. 
[16] Starikov E B and Pedash Y F 1990 Biopolymers 30 349-55.

[17] Aflatooni K, Gallup G A and Burrow P D 1998 J. Phys. Chem. A 102 6205-07.

[18] Russo N, Toscano M and Grand A 2000 J. Comput. Chem. 21 1243-50.

[19] Wesolowski S S, Leininger M L, Pentchev P N and Schaefer H F 2001 J. Am. Chem. Soc. 123 4023-28.

[20] Orlov V M, Smirnov A N and Varshavsky Y M 1976 Tetrahedron Lett. 4377-78.

[21] Hush N S and Cheung A S 1975 Chem. Phys. Lett. 34 11-13.

[22] Starikov E B 2003 Philos. Mag. Lett. 83 699-708.

[23] Brunaud G, Castet F, Fritsch A and Ducasse L 2002 Phys. Chem. Chem. Phys. 4 6072-79.

[24] Zhu Q Q and LeBreton P R 2000 J. Am. Chem. Soc. 122 12824-34.

[25] Kurita N and Kobayashi K 2000 Comput. Chem. 24 351-57.

[26] Andreoni W, Curioni A and Mordasini T 2001 IBM J. Res. Dev. 45 397-407.

[27] Ray S G, Daube S S and Naaman R 2005 Proc. Natl. Acad. Sci. U. S. A. 102 15-19.

[28] Voityuk A A, Jortner J, Bixon M and Rosch N 2000 Chem. Phys. Lett. 324 430-34.

[29] Yoo K H, Ha D H, Lee J O, Park J W, Kim J, Kim J J, Lee H Y, Kawai T and Choi H Y 2001 Phys. Rev. Lett. 87198102 (4pp).

[30] Correia H M G and Ramos M M D 2003 Mater. Sci. Eng. C-Biomimetic Supramol. Syst. 23 773-77.

[31] Brunaud G, Castet F, Fritsch A and Ducasse L 2003 Phys. Chem. Chem. Phys. 5 2104-13.

[32] Dedachi K, Natsume T, Nakatsu T, Ishikawa Y and Kurita N 2006 Int. J. Quantum Chem. 106 3270-77.

[33] Ullien D, Cohen H and Porath D 2007 Nanotechnology 18424015 (4pp). 